\title{
Towards understanding the etiology of high myopic strabismus using mechanical analysis and finite element modeling
}

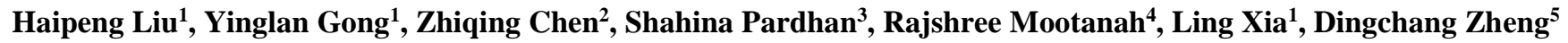 \\ 1.Department of Biomedical Engineering, Zhejiang University, Hangzhou, China, 310027
}

2.Eye Center, Second Affiliated Hospital, School of Medicine, Zhejiang University, Hangzhou, China

3.Vision \& Eye Research Unit, Postgraduate Medical Institute, Anglia Ruskin University, Cambridge, CB1 1PT, UK

4.Medical Engineering Research Group, Anglia Ruskin University, Chelmsford, CM1 1SQ, United Kingdom

5.Health \& Well Being Academy, Faculty of Medical Science, Anglia Ruskin University, Chelmsford, CM1 1SQ, UK

*Corresponding author

Dr.Yinglan Gong or Dr. Dingchang Zheng

E-mail: yinglangong@zju.edu.cn or Dingchang.zheng@anglia.ac.uk

Tel:+8613073668449

\begin{abstract}
It has been widely accepted that the pathology of high myopic esotropia, a special form of strabismus, is still not fully understood. In this study, the mechanical analysis and finite element analysis (FEA) of the oculomotor system was based on clinical MRI data and applied to examine the physiological hypotheses of extraocular muscle obliquity and deformation respectively. Our mechanical analysis indicated that the muscular obliquity is not the dominated cause of high myopic strabismus. Next, by simulating the effect of different forces applied to the cross section of each extraocular rectus muscles, the corresponding eyeball rotations were quantified on normal eyes, and high myopic eyes with and without strabismus. The model suggests that the limitation of rotation in high myopic strabismic eyes is mainly caused by the extraocular muscle deformation instead of, but related with ,its obliquity, providing a better understanding of the etiology of high myopic strabismus. To the best of our knowledge, this is the first mechanical and FEA model developed from clinical data to investigate the etiology of high myopic strabismus, providing important tools for future studies.
\end{abstract}

Keywords- eyeball; finite element analysis; high myopia strabismus; mechanical analysis; simulation

\section{INTRODUCTION}

The eyeball is held in place by three pairs of extraocular muscles, lateral rectus(LR) and medial rectus(MR) for horizontal movement, superior rectus(SR) and inferior rectus(IR) for vertical movement, and superior oblique(SO) and inferior oblique(IO) for rotation around the optical axis. High myopic strabismus happens in one or both eyes, with elongated eyeballs deformed and gradually anchored, finally resulting in strabismus fixus. This rare condition came into notice since the hypothesis of 'heavy eye syndrome' (Basghaw, 1966). However, the understanding of its etiology is still in process without definite conclusion.

With the development of advanced imaging technology, one etiological hypothesis was introduced based on the shift of muscle paths. With the paths of the rectus muscles gauged in high myopic patients using MRI imaging, it was found that the paths of all the 
muscles shifted obliquely in the wake of excessive axial elongation, with the inferior shifting of LR most obvious (Krzizok and Schroeder, 2003). Yoshiko et al also comprehensively described that a prolapsing eyeball shifts LR and SR nasally. The obliquity of muscles is particularly severe in patients with hypoesodeviation fixus. (Aoki et al., 2003). There was also a study negating the muscle obliquity as main etiology and intuitively emphasized the factor of muscle weakness(Hoerantner et al,2007), but the simulation was based on existing software with some clinical measurement, rather than totally on the clinical data. Latter clinical investigations confirmed the relationship between the path obliquity of extraocular muscles and high myopic esotropia(Demer,2010).

Another supposed etiology was about muscular deformation. In 1973, it was reported that the declination of the myofibers caused the deformation of extraocular muscles, especially in the LR (Duker-Elder and Wyber, 1973). In 1989, it was found that, the enlarged eyeball with posterior scleral staphyloma was pressed by the orbit, leading to deformed LR and inhibited eye movements (Demer and Noorden, 1989). With the establishment of the pulley theory which illuminated the stability of posterior extraocular muscle path (Demer et al., 1995), the restrictive dyskinesia is inferred to be a result of the displacement of the LR pulley. By measuring spontaneous action potentials of the MRs and LRs, Kong et al in 1995 (Kong et al., 1995) suggested palsy of deformed LR as the pathogenesis of high myopic strabismus.

In brief, the two hypothesized etiologies of high myopic strabismus lie respectively in the obliquity and deformation of extraocular muscles . For further validation, it is not available to perform related in-vivo clinical measurements, and the current imaging technology is also incapable of real-time observation of eyeball rotation with enough accuracy.

Fortunately, both mechanical analysis and finite element analysis (FEA) of the oculomotor system have the advantage of simulating the physiological movements without any destructive in-vivo tests. This method has been applied to achieve better understanding of eye structural properties (Schutte et al., 2006; Martin et al.,2005; Hermans et al.,2006), and the etiology and treatment of various ophthalmic diseases (Tse et al.,2012; Wang et al.,2000). In this paper, both methods were developed from clinical data, and then applied to simulate the eyeball movement respectively to understand the effect of muscle obliquity and deformation on the etiology of high myopic strabismus.

\section{MECHANICAL ANALYSIS: THE EFFECT OF MUSCLE OBLIQUITY ON STRABISMUS}

\section{A. Data source for the measurement of obliquity angle}

The eye dataset were from MRI imaging (SIEMENS/TrioTim, 3.0T) of a pair of normal eyes, and two pairs of high myopia eyes (one with and the other without bilateral strabismus) from Tianjin Medical University, China. The strabismic eyes have been clinically diagnosed using Hirschberg test, with bilateral $25^{\circ} \sim 30^{\circ}$ esotropia. Full ethical permission from Tianjin Medical University was obtained and the patients were consented before the measurement. The MRI images of both eyes from each patient were in DICOM format and of three directions. They were imported into the modeling software Mimics10.0. By using different masks, the eyeball, the extraocular muscles and the optic nerve were extracted respectively to form an intact 3-dimensional (3-D) eye model.

Prior to any further analysis on the 3-D eye model, it's necessary to confirm the effectiveness of the reconstructed model ensuring its consistency with real clinical conditions. According to Yokoyama’s judgement criterion (Yokoyama et al., 2001), the muscle obliquity can be accessed by the angle between the segments starting from the centroid of the eyeball to the controid of LR 
and SR. However, even a muscle has shifted sufficiently to deflect its force, the position of its junction with the eyeball may change little. Therefore, to show the obliquity more clearly, the measurement plane was moved posteriorly to the middle parts of the extraocular muscles, near the junction of eyeball and optic nerve. Our modified measurement was performed on the three pairs of eyeballs with the angles shown in Fig.1. The angle magnitude difference between strabismic and non-strabismic eyes is only obvious in right eyes, indicating that the measurement of obliquity angle is suggestive rather than conclusive since the patient was clinically diagnosed as bilateral strabismus.

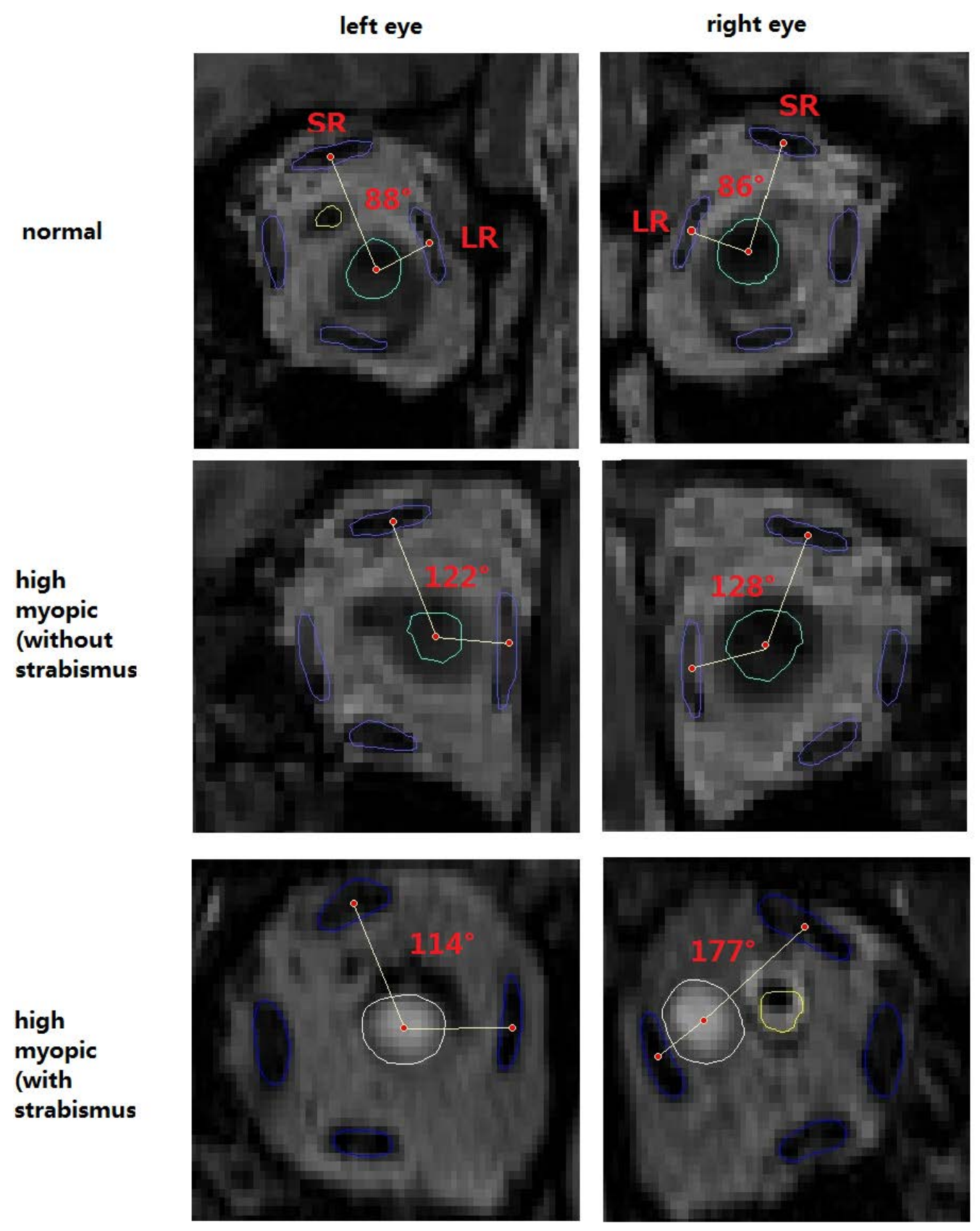

Figure 1. Angles between the lines from the centroid of the eyeball to the controid of LR and SR. The values of three pairs of eyes (normal), high myopic eyes without strabismus and with strabismus are given. 


\section{B. Mechanical analysis of oblique $L R$}

Using the angles measured from the images above, the strabismic LR obliquity, which is obvious in the right strabismic eye, can be derived in comparison with the eyes without strabismus. The obliquity angle in the posterior area of the eyeball is about $50^{\circ}$ (calculated from $177^{\circ}-128^{\circ}$ of the right myopic eyes with and without strabismus to investigate the effect of strabismus only). This obliquity is actually due to eyeball malposition, and can be deemed as caused by LR and SR equally, resulting in the derived LR obliquity angle of about $25^{\circ}$. However, the above example is an extremely strabismic case, and based on measurement in most deflected segment. For a more common example we consider a less severe obliquity (or deflection) angle of $10^{\circ}$.

According to prior knowledge and the published clinical data of Asian people, the modelled pulley of MR, LR, SR and IR starts from $4 \mathrm{~mm}, 8 \mathrm{~mm}, 6 \mathrm{~mm}$ and $6 \mathrm{~mm}$ posteriorly to the equatorial plane that splits the eyeball into anterior and posterior hemispheres equally (Fu et al., 2007). Since the posterior extraocular muscle are kept stable by pulley, the posterior muscle section keeps fixed and the force contraction of the whole muscle can be simulated by applying the force to the anterior section. Therefore in this model only the muscle sections anterior to the pulley, which rotate with the eyeball, were considered.

In order to confirm Yoshiko et al's supposition that the inferior shifting of the LR plays an important role in a fixed esotropia (Aoki et al., 2003), a simplified mechanical model is established on theoretical mechanics. As shown in Fig.2, the eyeball and the anterior sections of SR, MR and IR were simplified as a sphere and 3 parallel springs stretching backwards from the equatorial plane to the pulley plane respectively. The center of the sphere is fixed to exclude translation. The coordinate system was set by point $\mathrm{z}$ axis forward, $\mathrm{x}$ axis horizontally rightward and $\mathrm{y}$ axis vertically downward. The force on LR was exerted directly to the connection point on the equatorial plane of the strabismic eye. The direction of the force was deviated downward to simulate the LR obliquity, and was decomposed in y and $\mathrm{z}$ axes. Consider the component along y axis first. It causes eyeball rotation around the $\mathrm{z}$ axis. So if the $\mathrm{y}$ axis is attached to the eyeball, it will rotate to a new position, noted as ${ }^{y^{\prime}}$. The $\mathrm{z}$ axial component was then added, causing eyeball rotation around the $y^{\prime}$ axis.

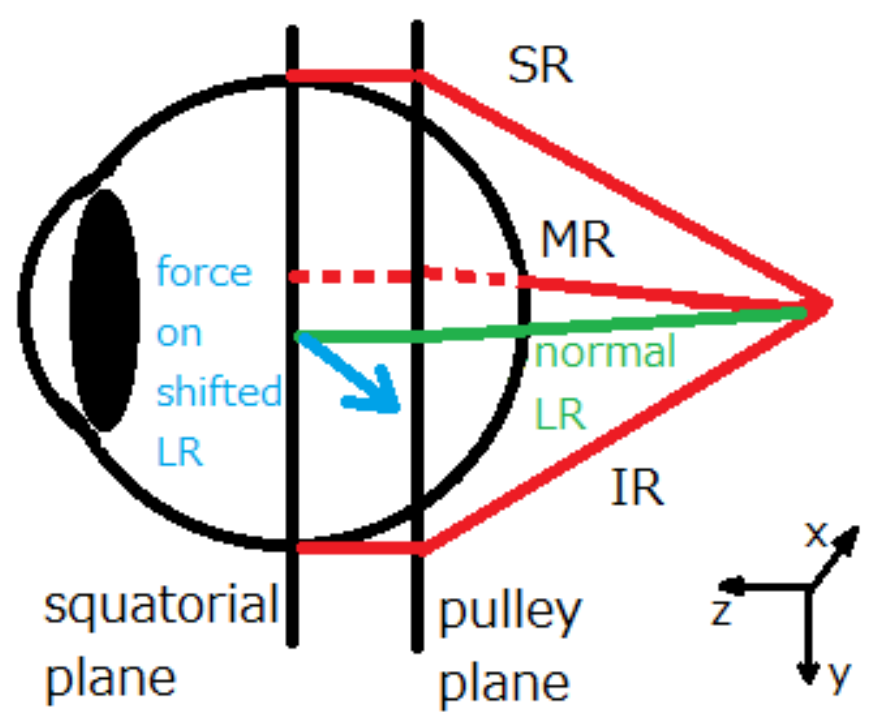

Figure 2. A simplified mechanical model of the eyeball and recti 
According to the final force equilibrium in the transverse and coronal planes(x-z plane and $x-y$ plane in the coordinate system), the relevant moment balance equations can be obtained respectively:

$$
\begin{gathered}
F_{y} \cdot R=2 k\left(\frac{d_{1}}{\cos \frac{R \theta}{d_{1}}}-d_{1}\right) \sin \frac{R \theta}{d_{1}} \cdot R+k\left(\sqrt{\left(d_{2}+\alpha R\right)^{2}+(R \theta)^{2}}-d_{2}\right) \cdot \frac{R \theta}{\sqrt{\left(d_{2}+\alpha R\right)^{2}+(R \theta)^{2}}} \cdot R \\
F_{z} \cdot R=k\left(\sqrt{\left(d_{2}+\alpha R\right)^{2}+(R \theta)^{2}}-d_{2}\right) \cdot \frac{d_{2}+\alpha R}{\sqrt{\left(d_{2}+\alpha R\right)^{2}+(R \theta)^{2}}} \cdot R
\end{gathered}
$$

The downward component of the LR force is supposed to rotate the eyeball around the $\mathrm{z}$ axis firstly, and then the eyeball rotates around the current y' axis, so the whole process can be decomposed and expressed with coordinate transformation matrix equations as follow:

$$
\begin{gathered}
{\left[\begin{array}{l}
x_{1} \\
y_{1} \\
z_{1}
\end{array}\right]=\left[\begin{array}{ccc}
\cos \theta & -\sin \theta & 0 \\
\sin \theta & \cos \theta & 0 \\
0 & 0 & 1
\end{array}\right] \cdot\left[\begin{array}{l}
x \\
y \\
z
\end{array}\right]} \\
{\left[\begin{array}{l}
x_{1}^{\prime} \\
y_{1}^{\prime} \\
z_{1}^{\prime}
\end{array}\right]=\left[\begin{array}{l}
x \\
y \\
z
\end{array}\right]}
\end{gathered}
$$

$$
\left[\begin{array}{l}
x_{2}^{\prime} \\
y_{2}^{\prime} \\
z_{2}^{\prime}
\end{array}\right]=\left[\begin{array}{ccc}
\cos \alpha & 0 & -\sin \alpha \\
0 & 1 & 0 \\
\sin \alpha & 0 & \cos \alpha
\end{array}\right]\left[\begin{array}{l}
x_{1}^{\prime} \\
y_{1}^{\prime} \\
z_{1}^{\prime}
\end{array}\right]
$$

The meanings of the notations are listed in Table 1.

TABLE I. NOTATIONS OF THE PARAMETERS USED IN THE EQUATIONS AND THEIR MEANINGS

\begin{tabular}{|l|l|}
\hline \multicolumn{1}{|c|}{ Notation } & \\
\hline$F_{y}$ & Force component in y axis. \\
\hline$F_{z}$ & Force component in z axis. \\
\hline$d_{1}$ & The distance from the fixed point of SR and IR to their pulleys. \\
\hline$d_{2}$ & The distance from the fixed point of MR to its pulley. \\
\hline $\mathrm{R}$ & Eyeball radius. \\
\hline $\mathrm{a}$ & Radians of rotation around y' axis(horizontal rotation angle). \\
\hline
\end{tabular}




\begin{tabular}{|c|c|}
\hline$\theta$ & Radians of rotation around $\mathrm{z}$ axis(downward deflection angle). \\
\hline $\mathrm{k}$ & Stiffness of the spring \\
\hline$\left(\begin{array}{lll}x & y & z\end{array}\right)^{T}$ & Coordinates in the initial coordinate system \\
\hline$\left(\begin{array}{lll}x_{1} & y_{1} & z_{1}\end{array}\right)^{T}$ & $\begin{array}{l}\text { Coordinates in the initial coordinate system } \\
\text { after rotation around } \mathrm{z} \text { axis. }\end{array}$ \\
\hline$\left(\begin{array}{lll}x_{1}^{\prime} & y_{1}^{\prime} & z_{1}^{\prime}\end{array}\right)^{T}$ & Coordinates in the changed coordinate system after rotation around $\mathrm{z}$ axis. \\
\hline$\left(\begin{array}{lll}x_{2}^{\prime} & y_{2}^{\prime} & z_{2}^{\prime}\end{array}\right)^{T}$ & Coordinates in the changed coordinate system after rotation around $\mathrm{z}$ axis and $y^{\prime}$ axis \\
\hline$\left(\begin{array}{lll}x_{2} & y_{2} & z_{2}\end{array}\right)^{T}$ & Coordinates in the initial coordinate system after rotation around $\mathrm{z}$ axis and $y^{\prime}$ axis \\
\hline
\end{tabular}

By exploiting Taylor expansion with the higher order infinitesimals ignored, the expression of $\alpha$ and $\theta$ are derived from (1) and (2):

$$
\begin{gathered}
\alpha=\frac{F_{z}}{k R} \\
\theta=\frac{F_{y} d_{2}}{F_{z} R}
\end{gathered}
$$

And from (3), (4) and (5) the final coordinate transformation is obtained:

$$
\left[\begin{array}{l}
x_{2} \\
y_{2} \\
z_{2}
\end{array}\right]=\left[\begin{array}{ccc}
\cos \theta & -\sin \theta & 0 \\
\sin \theta & \cos \theta & 0 \\
0 & 0 & 1
\end{array}\right]\left[\begin{array}{ccc}
\cos \alpha & 0 & -\sin \alpha \\
0 & 1 & 0 \\
\sin \alpha & 0 & \cos \alpha
\end{array}\right]\left[\begin{array}{l}
x \\
y \\
z
\end{array}\right]
$$

Substituting (6) and (7) into (8), the final position of any point on the spherical surface can be solved.

If $\sqrt{F_{z}^{2}+F_{y}^{2}}=k \cdot R \cdot \frac{\pi}{30}$ ( the undeflected force on LR rotates the eye $10^{\circ}$ horizontally, as a common case ) and $F_{y}=F_{z} \cdot \tan 10^{\circ}$ (here the $10^{\circ}$ is the force deflection angle from the Yokoyama measurement analysis above), according (6) and (7), the obtained horizontal rotation angle $(\alpha)$ and downward deflection angle $(\theta)$ of eyeball were only $9.8^{\circ}$ and $1.7^{\circ}$ respectively.

It could be concluded that the effect of pulley obliquity alone is insufficient to explain the radical change of the eyeball position, since downward deflection angle of a common strabismic eyeball often is often over 10 (Kong et al., 1995; Yokoyama et al.,2001; Yamaguchi et al.,2010). 


\section{CONSTRUCTION AND VALIDATION OF FEA MODEL}

\section{A. Construction of FEA model}

In order to polish the primitive 3-D model, it was further processed with GeomagicStudio12.0 to be smoothed, with some erroneous details amended, as shown in Fig.3. It was then input into the CAD software Solidworks2010 to make final shape modifications, and finally input into Solidworks Simulation as an assembly to perform FEM analysis.

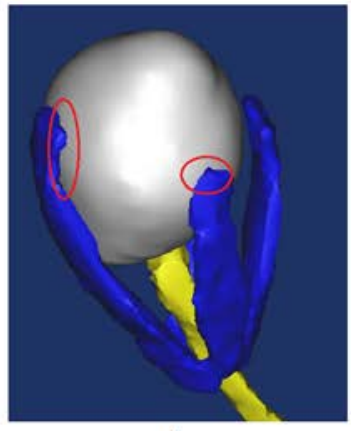

A

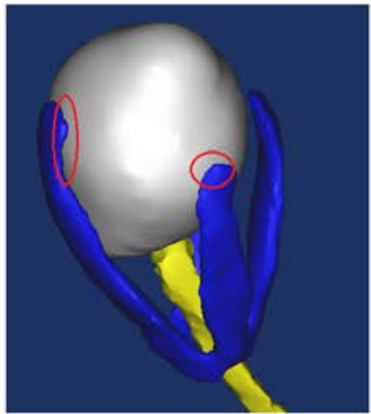

B

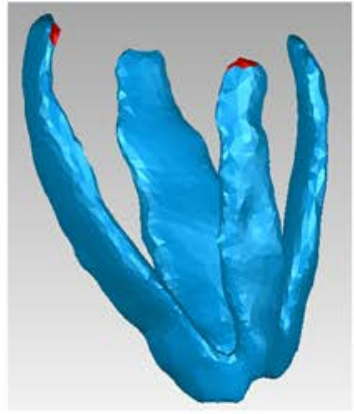

C

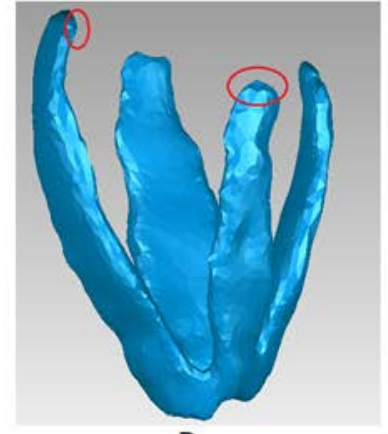

D

Figure 3. Polishing and processing of the eye model. (A)\&(B)The model in the Mimics before and after smoothing. (C)\&(D)The extraocular muscles from Mimics are checked in Geomagics to make some minor amendments.

To perform the FEM analysis, the properties of related tissue materials (including eyeball, extraocular muscle and tendon) were stipulated. The material properties from normal eyes were obtained from published clinical data (Zhou et al., 2006; Man,1995; Jouzdani and Sara,2013), as given in Table 3. The muscle tendons that connect the recti and the eyeballs were simulated with spring elements. The eyeball was constricted to rotate around its center. The pulley can be regarded as a smooth socket which constricts the translation in the normal direction of the surface. By constricting the normal translation of the pieces, as shown in the green arrows in Fig.4. With muscle path direction unchanged, the physiologic function of pulley was therefore intimated. Forces will be imposed in the following simulation to verify the model.

TABLE II. THE MATERIAL PROPERTIES OF DIFFERENT TISSUES.

\begin{tabular}{|l|c|c|c|c|}
\hline \multicolumn{1}{|c|}{$\begin{array}{c}\text { Materials } \\
\text { Properties }\end{array}$} & $\begin{array}{c}\text { Elastic Modulus } \\
\left(N / m^{2}\right)\end{array}$ & Poisson's Ratio & $\begin{array}{c}\text { Tangential Stiffness } \\
(N / m)\end{array}$ & $\begin{array}{c}\text { Axial Stiffness } \\
(\mathrm{N} / \mathrm{m})\end{array}$ \\
\hline Eyeball & $3,000,000$ & 0.3 & & \\
\hline Extraocular muscle & 82,000 & 0.4 & & 16,000 \\
\hline Tendon & & & 83,000 & \\
\hline
\end{tabular}

\section{B. Model verification on strabismic eye model in comparison with published model}

According to Lancaster's computational theory, 1-1.75g $(0.01 \mathrm{~N}-0.018 \mathrm{~N})$ force is sufficient to make eyeball rotate (Zhou et al., 2006). A pair of $0.03 \mathrm{~N}$ forces applied to the anterior and posterior poles of the eyeball respectively in opposite directions could form a force couple with a the theoretical rotation value of the eyeball between $7^{\circ}$ and $10^{\circ}$ (Zhou et al., 2006). The adduction and abduction of both eyeballs were therefore simulated. In vertical directions, force couples with the same force magnitude were also applied to simulate the supraduction and infraduction. Results converge well, and are listed in Table 3. 
TABLE III.

THE FEA RESULTS OF ARTIFICIAL EYEBALL ROTATION FROM OUR MODEL RESULTS IN COMPARISON WITH PUBLISHED DATA.

\begin{tabular}{|l|l|l|l|l|}
\hline $\begin{array}{c}\text { Our } \\
\text { Values/Zhou et } \\
\text { al's }\end{array}$ & $\begin{array}{c}\text { Rotation of the } \\
\text { eyeball }\end{array}$ & $\begin{array}{c}\text { Maximal Node } \\
\text { Displacement } \\
(\mathbf{m m})\end{array}$ & $\begin{array}{c}\text { Maximal Node } \\
\text { Von Mises } \\
\text { Stress(kPa) }\end{array}$ & $\begin{array}{l}\text { Maximal Node } \\
\text { Strain(ESTRN) }\end{array}$ \\
\hline Adduction & $7.3^{\circ} / 9.1^{\circ}$ & $1.5 / 3.8$ & $340 / 49$ & $0.9 / 0.8$ \\
\hline Abduction & $8.2^{\circ} / 8.2^{\circ}$ & $1.7 / 3.5$ & $368 / 44$ & $1.0 / 0.8$ \\
\hline Supraduction & $7.3^{\circ} / 8.7^{\circ}$ & $1.5 / 2.4$ & $349 / 27$ & $0.7 / 0.5$ \\
\hline Infraduction & $7.4^{\circ} / 7.9^{\circ}$ & $1.6 / 2.2$ & $349 / 24$ & $0.6 / 0.4$ \\
\hline
\end{tabular}

Our results in Table 3 were compared with the counterparts from Zhou et al's. (Zhou et al., 2006). Although in our model eyeball rotations were smaller, the differences were acceptable. However, our stress results were larger in about an order of magnitude. The difference possibly lies in the difference of the tendon simulation. The eyeballs in their model were directly bonded with muscles while we used spring elements to simulate the tendons. The stress concentration at the joint points would account for higher stress magnitude. Tendon stiffness is larger compared with muscle, so resistance in our model was larger with smaller rotation degrees, which is closer to the physiological situation.

By comparison above, our FEM model has been validated for further analysis. With similar method, the FEM models of high myopic eyes with and without strabismus were built.

\section{FEA SimUlation: THE EFFECT OF MusCle DeFormation ON STRABISMUS}

Force with varied magnitude from $0.03 \mathrm{~N}, 0.05 \mathrm{~N}, 0.07 \mathrm{~N}, 0.08 \mathrm{~N}$ to $0.1 \mathrm{~N}$ was applied on the cross section of each extraocular recti respectively. Force was applied on only one muscle once, with other three recti constrained. The functional relationship between the degrees of rotation and the magnitude of the force was obtained for each muscle.

Fig. 4 demonstrates one example with $0.1 \mathrm{~N}$ force applied on LRs of normal eyes, high myopic eyes without strabismis, and high myopic eyes with strabismus. The angle between the dotted yellow line(the direction of SR, which moves little in the horizontal rotation of the eyeball) and the red line(the direction of optic axis, or the diameter between anterior and posterior poles of the eyeball) shows the consequent rotation. So it is obvious that the strabismic eyes hardly rotate with force, compared with the normal eyes and the high myopic eyes without strabisums. 


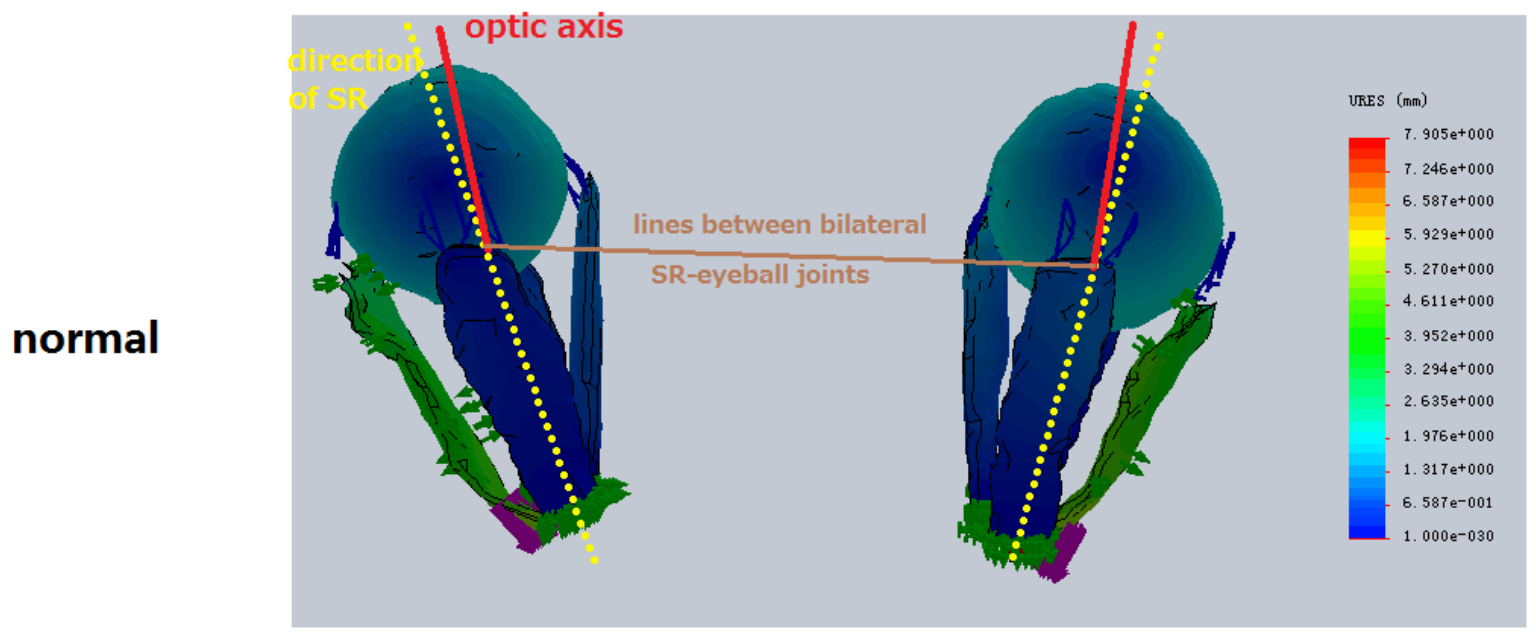

high myopic (without strabismus)

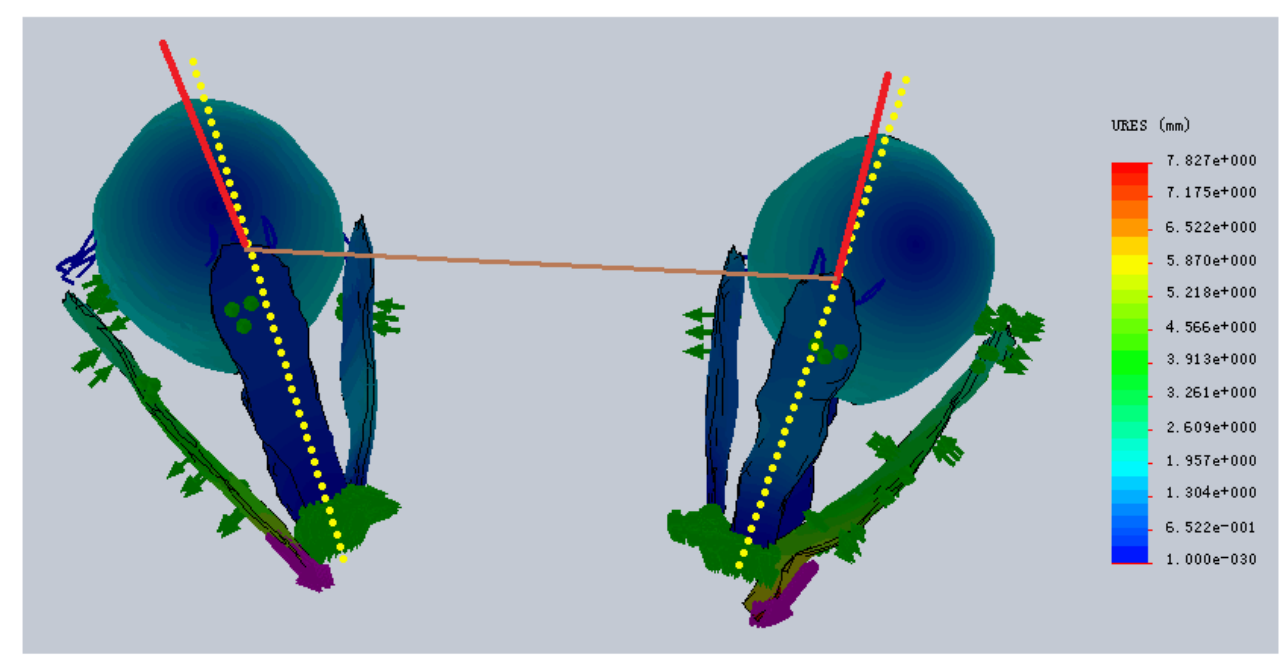

high myopic (with strabismus)

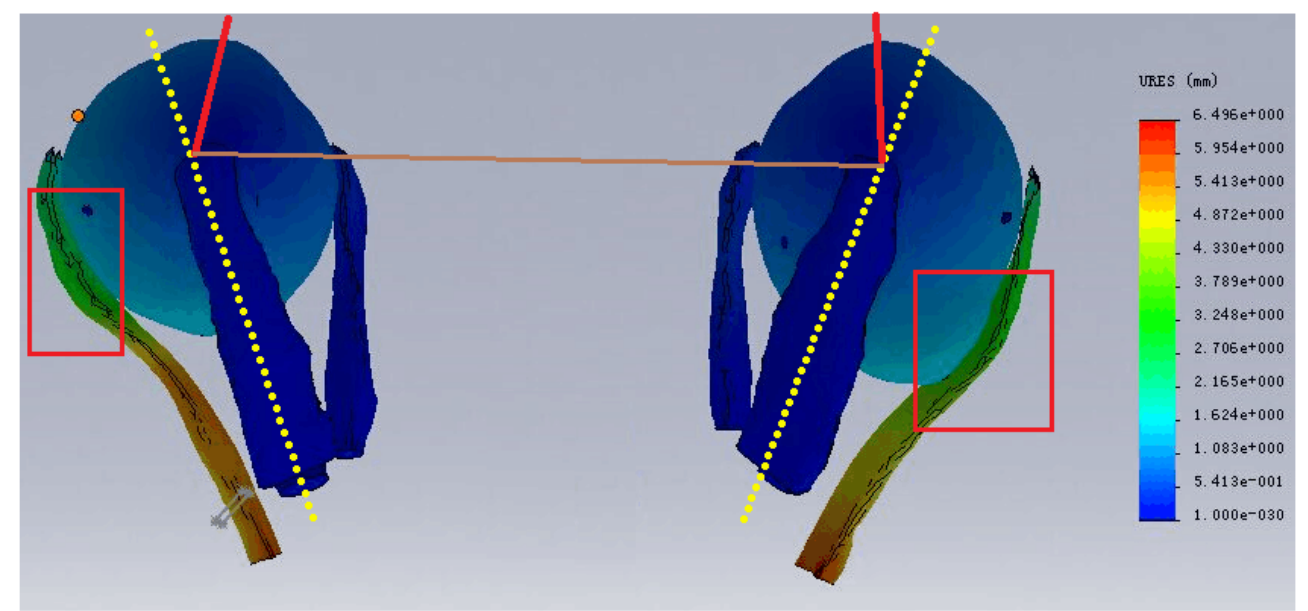


Figure 4. Eyeball displacement with $0.1 \mathrm{~N}$ force applied on LR. Red lines: direction of optic axis. Brown lines: the connection between left and right joint of SR and eyeball. Yellow dotted lines: SR direction. Red rectangles: the areas where eyeball and LR contact.

The different rotation magnitude validates the necessity to investigate the force-rotation relationship. As shown in Fig.5, the displacements at different points along the horizontal great circle (not an exact circle due to the deformation of myopic eyeball) perpendicular to the rotating vectors were measured and then averaged. The rotation angle was then calculated from the geometric relationship with known eyeball radius, as shown in Fig.5.

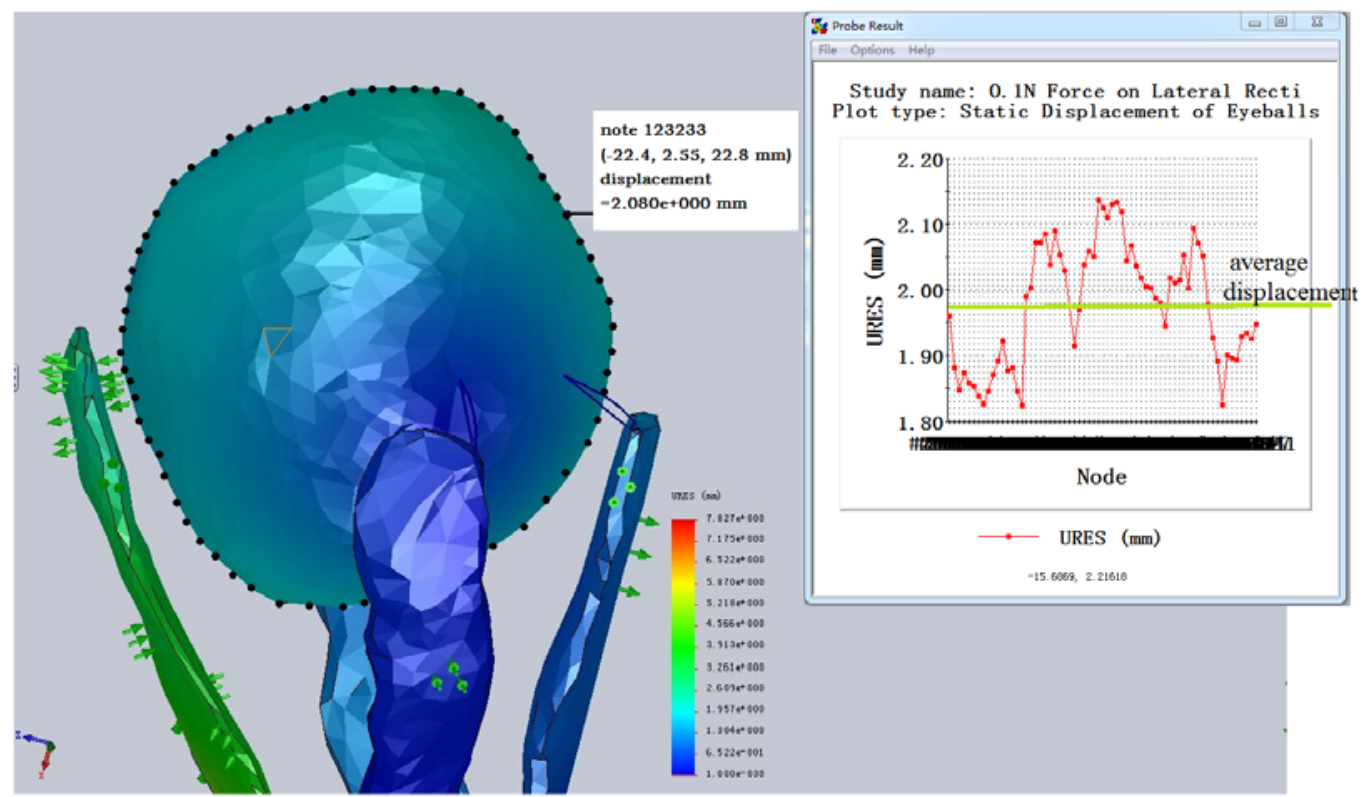

A: Measurement of displacement along the big circle and take the average
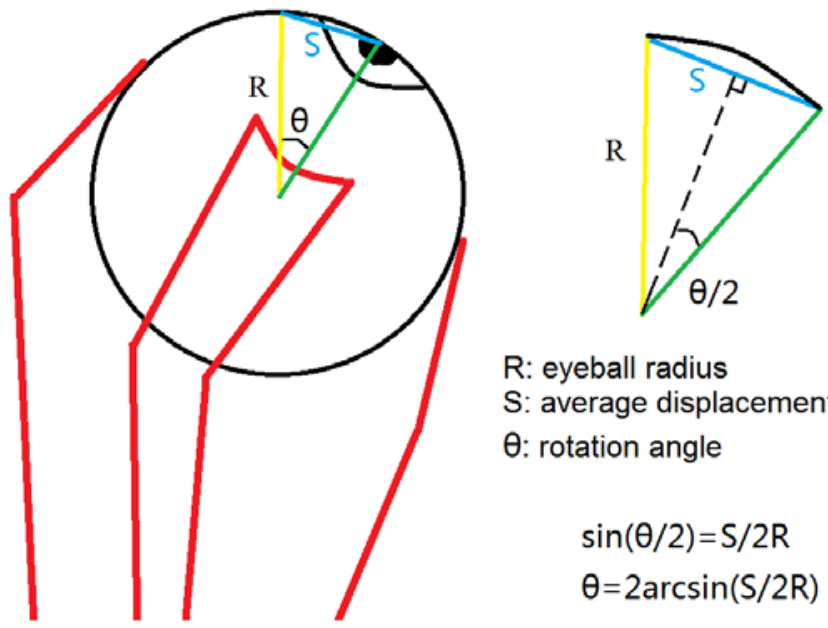

R: eyeball radius

$\mathrm{S}$ : average displacement along the great circle

$\theta$ : rotation angle

$$
\begin{aligned}
& \sin (\theta / 2)=S / 2 R \\
& \theta=2 \arcsin (S / 2 R)
\end{aligned}
$$

\section{B: Calculation of the rotation angle using displacement}

Figure 5. (A)The displacement of the eyeball is measured(result of one point is shown as an example) along the great circle and then averaged (B)The angle of rotation is calculated using anti-trigonometric function. 
Fig. 6 shows the eyeball rotation caused by LR and MR in the three pairs of eyes respectively. The relationship between the applied forces and rotations are basically linear, which is reasonable for small-angle rotations of eyeball. Some traits can be derived.

Firstly, all horizontal rotations in strabismic eyes are obviously weakened, compared with normal eyes and high myopic eyes without strabismus. During the rotation, the strabismic eyeball contacts with the LR, as shown in the red rectangles in Fig.4. This explains the movement limitation in strabismic eyes, suggesting that the deformation and malposition of the prolapsed eyeball constrains the eyeball movement.

Secondly, in strabismic eyes, rotations caused by LRs are further constrained than those caused by MRs. This could be explained by the weakened LRs due to prolonged squeezing between the elongated eyeball and the orbit, suggesting that the LR weakness may be an important factor.

Finally, lateral difference exists in strabismic eyes. The curves of both MRs lie in proximity, while the right LR curve lies apparently lower than the left one. Related shape difference can be obverved. In Fig.4, the right eyeball is with severe posterior scleral staphyloma and deformed LR thinner than its left counterpart. The stress concentration also happens in these thinned areas.

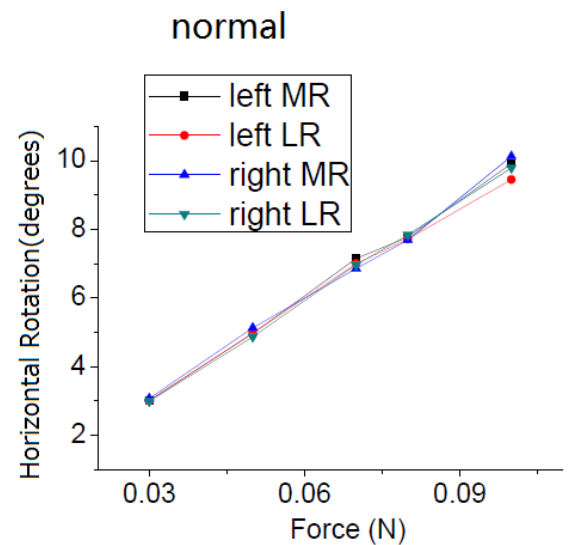

high myopic(without strabismus)

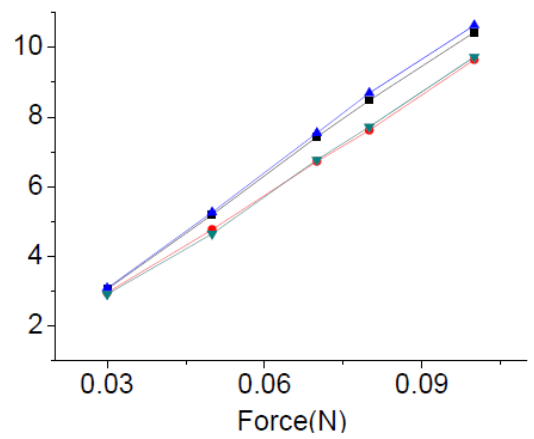

high myopic(with strabismus)

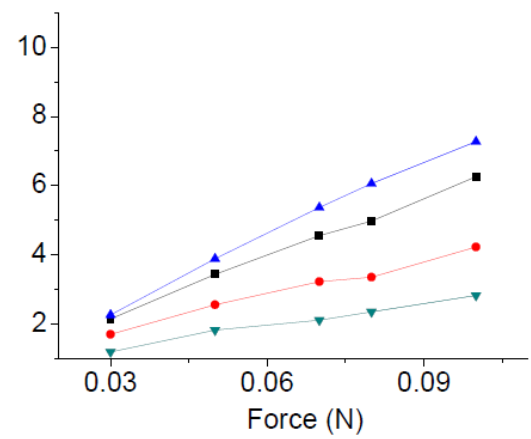

Figure 6. The functional relation between force and horizontal rotation for LRs and MRs of the normal eyes, highly myopic eyes, and eyes with highly myopic strabismus. The results for both left and right eyes are given.

Fig.7 shows the relationship between vertical rotation and the forces on SR and IR. Firstly, similar with horizontal rotation, the relationships are linear, an overall strabismic movement limitation is obvious. Secondly, in all the cases, the left/right differences are ignorable with supraduction always slightly smaller than infraduction. In terms of muscle configuration, both width and thickness of the IRs are larger than those of SRs in all cases. This phenomenon may be related with human view habits of looking more downward than upward. The SR/IR difference is more obvious in the strabismic eyes which may partly explain the reason for hypotropia. 

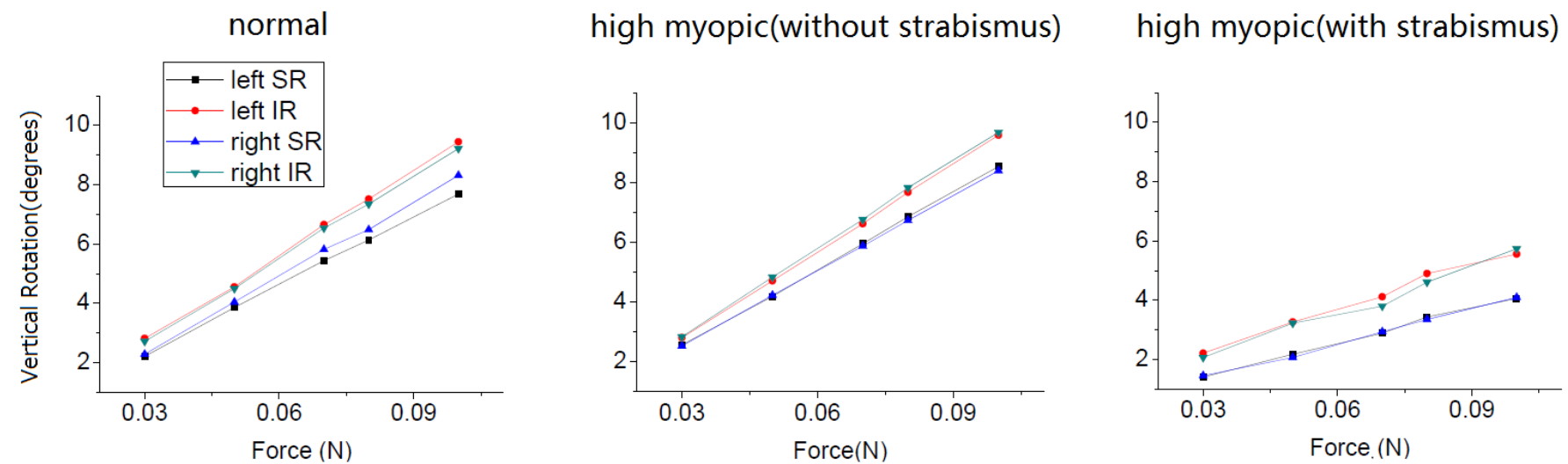

Figure 7. The relationship between force and vertical rotation for SRs and IRs of the eyes of the normal eyes, highly myopic eyes, and eyes with highly myopic strabismus. The results for both left and right eyes are given.

In our models of normal eyes and high myopic eyes, the rate between rotation and force changes was within the range 0.008N/deg 0.011N/deg. The corresponding rate from Gao et al’s model was 0.011N/deg (Gao and Chen, 2014), and the clinical measurement from Collins et al. was 0.0084N/deg 0.010N/deg (Collins et al., 1981). Therefore, our simulation results agreed with the clinical measurement and results from published models.

\section{DISCUSSION}

Our results of mechanical analysis and FEA revealed the complex relationship between muscle deflection and deformation in eyes with high myopic strabismus.Briefly, the limitation of rotation in strabismic eyes is caused by the combination of extraocular muscle deformation and obliquity,with the former as the main factor. The suggestive conclusion in simulation study(Hoerantner et al,2007) is confirmed here with clinical data. Severe weakness of the LR could be one of the main causes of high myopic esotropia. It can be inferred that muscular weakness mainly stems from the deformed shape due to lasting contact with the elongated eyeball. This has been confirmed by the clinical finding that the postoperative rehabilitation is progressive process that lasts for months (Wong et al., 2005; Ahadzadeghan et al., 2009), as it takes some time for a muscle to adjust its configuration and length(Goldspink and Williams, 1992). The instant postoperative relief should be owed to the correction of the eyeball and muscle path, and the slow final recovery from strabismus is related to the growth and recovery of the corresponding extraocular muscles. It has been confirmed that extraocular muscle can adjust their lengths throughout life by the addition or subtraction of sarcomeres for optimal function over the range that they are used (Goldspink and Williams, 1992).

Our modeling approach could also be beneficial for the treatment of high myopic strabismus (Sturm et al., 2008). Based on the understanding of its etiology (Yokoyama et al., 2001) and accumulating experience of traditional operation using rectus recession and resection(Michael et al., 2014), the surgical operation proposed by Yokoyama et al (Yokoyama et al., 2001) has been proved efficient, which was later modified (Mishra, 2014; Akar et al., 2014; Kekunnaya et al., 2013). In the future, the surgical operation could be simulated with more clinical data and improved modeling approaches.

However, there are some limitations which need to be stated on our development of mechanical analysis and FEA modelling. Firstly, deviation exists within the displacement measurement process. For example, the rotation angle depends on the average displacement in the plane perpendicular to the axis of rotation. The coarseness and deformation of the eyeball model will slightly affect the results. This partially explains the disturbed linearity of force-rotation functions of the strabismic eyes. Secondly, the 
simplifications in the process inevitably sacrificed the accuracy of simulation. For example, to simulate the pulley, in certain areas (certain pieces of the triangle-facet mesh) muscles are limited to move along its surface without any normal motion. But the pieces are chosen manually, and the normal motion was not totally excluded under physiological conditions. This could be improved in a future model to which orbital fat is added in order to substitute the mechanical constraints. In addition, the material properties of the different tissues used in our model were from normal eyes. Their values could be different in high myopic eyes. Finally, the whole process of eyeball deformation and shifting with muscle distortion needs further study based on more advanced measurement technology. Clinical data can make further confirmation of our conclusions and polish the model with more precise parameters.

In summary, to the best of our knowledge, this is the first mechanical and computing model based on clinical data in the field of high myopic strabismus. Our modelling results are consistent with the clinical measurements and other published models, demonstrating the effectiveness of our model. Our modeling approach overcomes the difficulty of in-vivo measurement and cast lights on the etiology study of high myopic strabismus in terms of eye movement analysis.

\section{References}

[1] J Basghaw - Br J,The 'heavy eye' phenomenon. A preliminary report. Ophthalmol 1966

[2] TH Krzizok, BU Schroeder, Quantification of recti eye muscle paths in high myopia Strabismus, 2003

[3] Yoshiko Aoki, Yasuhiro Nishida, Osamu Hayashi, Jiro Nakamura, Sanae Oda, Shinichi Yamade, Kazutaka Kani.Magnetic resonance imaging measurements of extraocular muscle path shift and posterior eyeball prolapse from the muscle cone in acquired esotropia with high myopia.American Journal of Ophthalmology Volume 136, Issue 3, September 2003, Pages 482-489

[4] Hoerantner R, Kaltofen T, Priglinger S, et al. Model-based improvements in the treatment of patients with strabismus and axial high myopia[J]. Investigative Ophthalmology and Visual Science, 2007, 48(3): 1133.

[5] Demer JL. Editorial: Muscle paths matter in strabismus associated with axial high myopia. American journal of ophthalmology. 2010;149:184.

[6] Duker-Elder,Wyber K. System of Ophthamology. Ocular Motility Strabismus ,1973;6(3):607-608

[7] Demer JL, Noorden JK. High myopia as an unusual cause of restrictive motility disturbance. Surv Ophthamol ,1989;33(4):281-284

[8] Demer JL, Miller JM, Poukens V, et al. Evidence for fibromuscular pulleys of the recti extraocular muscles[J]. Investigative Ophthalmology \& Visual Science,1995, 36(6): 1125-1136.

[9] Kong Ling-yuan, et al. The characteristics and etiology of esotropia fixus with high myopia Chinese Journal of Strabismus \& Pediatric Ophthalmology 1995;2(3):64-65

[10] Schutte S, Sp V D B, Van K F, et al. A finite-element analysis model of orbital biomechanics.[J]. Vision Research, 2006, 46(11):1724-1731.

[11] Martin H. Comparison of the accommodation theories of Coleman and of Helmholtz by finite element simulations.[J]. Vision Research, 2005, 45(22):29102915.

[12] E.A. Hermans, M. Dubbelman, G.L. van der Heijde, et al. Estimating the external force acting on the human eye lens during accommodation by finite element modelling.[J]. Vision Research, 2006, 46(21):3642-3650.

[13] Tse K M, Lee H P, Shabana N, et al. Do shapes and dimensions of scleral flap and sclerostomy influence aqueous outflow in trabeculectomy? A finite element simulation approach[J]. British Journal of Ophthalmology, 2012, 96(3):432-437.

[14] Wang J Q, Zeng Y J, Li X Y. Influence of some operational variables on the radial keratotomy operation[J]. British Journal of Ophthalmology, 2000, 84(6):651-653.

[15] Yokoyama T, Ataka S, Tabuchi H, et al. Treatment of progressive esotropia caused by high myopia-a new surgical procedure based on its pathogenesis[C]//Transactions: 27th Meeting, European Strabismological Association, Florence, Italy. 2001: 145-148.

[16] Fu Te ,Wang Li-Hua,YU Tai-fe.The study of the location of human rectus pulleys on ocular dynamic MRI .Chin JPractOphthalmol, November2007, Vol25, No. 11 
[17] Yamaguchi M, Yokoyama T, Shiraki K. Surgical procedure for correcting globe dislocation in highly myopic strabismus[J]. American journal of ophthalmology, 2010, 149(2): 341-346. e2.

[18] Yiyi, Zhou, et al. "Finite Element Modeling and Biomechanical Analysis of Eyeball and Extraocular Muscles." Engineering in Medicine and Biology Society, 2005. IEEE-EMBS 2005. 27th Annual International Conference of the. IEEE, 2006.

[19] Hou Man.Muscular elasticity and its measurement.Journal of Beijing University of Physical Education.1995, 18(2): 41-45.

[20] Jouzdani, Sara. Biomechanical characterization and computational modeling of the anterior eye. Diss. UNIVERSITY OF MINNESOTA, 2013.

[21] GAO Zhi-peng, CHEN Wei-yi. Pulley tissues maintain the mechanical advantage of extraocular muscles under eye adduction: A simulation study.[J]. Journal of Medical Biomechanics(Yiyong Shengwu Lixue), 2014, 29(006): 498-503.

[22] Collins C C, Carlson M R, Scott A B, et al. Extraocular muscle forces in normal human subjects[J]. Investigative ophthalmology \& visual science, 1981, 20(5): 652-664.

[23] Wong I, Leo S W, Khoo B K. Loop myopexy for treatment of myopic strabismus fixus[J]. Journal of American Association for Pediatric Ophthalmology and Strabismus, 2005, 9(6): 589-591.

[24] Ahadzadeghan I, Akbari M R, Ameri A, et al. Muscle belly union for treatment of myopic strabismus fixus[J]. Strabismus, 2009 , 17(2): 57-62.

[25] Goldspink G, Williams P. Cellular mechanisms involved in the determination of muscle length and mass during growth; problems arising from imbalance between antagonists muscle groups[C]//Proceedings of the mechanics of strabismus symposium. 1992: 195-206.

[26] Sturm V, Menke M N, Chaloupka K, et al. Surgical treatment of myopic strabismus fixus: a graded approach[J]. Graefe's Archive for Clinical and Experimental Ophthalmology, 2008, 246(9): 1323-1329.

[27] Michael G, Birgit L, et al. Recess-resect surgery with myopexy of the lateral rectus muscle to correct esotropia with high myopia . British Journal of Ophthalmology, 2014-306429 Published Online First: 18 June 2015 doi:10.1136/bjophthalmol-2014-306429

[28] Mishra C. Modified 'Yokoyama Surgery ‘For’Heavy Eye Syndrome’[J].

[29] Akar S, Gokyigit B, Aribal E, et al. Surgical Procedure Joining the Lateral Rectus and Superior Rectus Muscles With or Without Medial Rectus Recession for the Treatment of Strabismus Associated With High Myopia[J]. Journal of Pediatric Ophthalmology \& Strabismus, 2014, 51(1): 53.

[30] Kekunnaya R, Shenoy H B, Sachdeva V. Silicone band loop myopexy in treatment of myopic strabismus fixus: Surgical outcome of a novel modification[J]. Journal of American Association for Pediatric Ophthalmology \& Strabismus, 2013, 17(1):e6. 